\title{
Análisis de las consecuencias del comportamiento adaptativo individual sobre la estabilidad poblacional: El caso del forrajeo óptimo
}

\author{
Analysis of the consequences of individual adaptive behavior on population stability: The \\ case of optimal foraging
}

\author{
FERNANDA S. VALDOVINOS $1,2,{ }^{*}$, PASQUINELL URBANI ${ }^{1} \&$ RODRIGO RAMOS-JILIBERTO ${ }^{1,2}$ \\ ${ }^{1}$ Centro Nacional del Medio Ambiente, Fundación de la Universidad de Chile, Av. Larraín 9975 La Reina, Santiago, Chile \\ ${ }^{2}$ Facultad de Ciencias, Universidad de Chile, Las Palmeras 3425 Ñuñoa, Santiago, Chile \\ *Autor correspondiente: fevaldovinos@gmail.com
}

\begin{abstract}
RESUMEN
Trabajos tempranos basados en la teoría de Sistemas Dinámicos, demostraron que mientras mayor sea el número de poblaciones en interacción ecológica, el sistema tiende a ser más inestable. Sin embargo, la evidencia empírica indica que las poblaciones naturales presentan más frecuentemente dinámicas estables, aun estando inmersas en comunidades complejas. Entre los mecanismos que promueven la estabilización poblacional, se encuentra el comportamiento adaptativo de los individuos componentes de las poblaciones. En este trabajo se analizan los avances teóricos respecto del rol del forrajeo óptimo (FO) como fuerza estabilizadora de las dinámicas poblacionales, en comunidades modelo de diferente nivel de complejidad estructural. El análisis se organiza en torno a tres preguntas centrales: i) ¿cuál es el sistema control contra el cual se compara la estabilidad de una población cuyos individuos presentan FO?, ii) ¿cuál es el concepto de estabilidad utilizado? y iii) ¿cómo se incorporan los supuestos del FO en las reglas que gobiernan la dinámica poblacional? En base a este análisis, especificamos los puntos que deben atenderse para evaluar correctamente el rol estabilizador del FO y otros comportamientos adaptativos que satisfagan los supuestos de la Teoría Ecológica de la Optimización. Finalmente, conjeturamos que el efecto estabilizador del FO dependerá cualitativamente tanto del nivel de recursos en el sistema, como de la relación entre tasa de perturbación ambiental y tasa de adaptación del depredador.
\end{abstract}

Palabras clave: comportamiento adaptativo, dieta, modelos poblacionales, optimización, plasticidad fenotípica.

\begin{abstract}
Early work based on the Dynamical Systems Theory demonstrates that the larger the number of interacting populations, the system tends to be more unstable. Nevertheless, empirical evidence indicates that natural populations more often exhibit stable dynamics, in spite of being embedded into complex communities. Adaptive behavior of individuals is found to be one of the mechanisms promoting population stabilization. In this work, we analyze the theoretical advances about the role of optimal foraging (FO) as a stabilizing force of population dynamics, in model communities with different levels of structural complexity. Our analysis is organized around three central points: i) what is the control system against which it is compared the stability of a population whose indviduals exhibit FO?, ii) what stability concept is being used?, and iii) how the assumptions of FO are incorporated within the rules governing the dynamics of populations? Based on our analysis, we specify the points that should be addressed for evaluating properly the stabilizing role of FO, as well as other kinds of adaptive behavior that satisfy the assumptions of the Ecological Optimization Theory. Finally, we conjecture that the stabilizing effect of FO will be qualitatively dependent on the level of resources in the system, and the ratio of environmental perturbation rate to the predator's adaptation rate.
\end{abstract}

Key words: adaptive behavior, diet, optimization, phenotypic plasticity, population models.

\section{INTRODUCCIÓN}

La coexistencia de especies en sistemas naturales y la estabilidad de los patrones de cambio temporal en el tamaño de las poblaciones, han sido tópicos centrales en el desarrollo de la teoría ecológica contemporánea (McCann 2000). A partir del trascendental trabajo de May (1972), la creencia temprana de que comunidades más diversas serían más estables (McArthur 1955, Hutchinson 1959) perdió su hegemonía. May (1972) se basa en la teoría matemática de sistemas dinámicos para demostrar que mientras mayor sea el número de poblaciones en interacción, el sistema tiende a ser más inestable. Este resultado descansa en el supuesto que no existen restricciones en la 
arquitectura de las interacciones entre especies, ni en las magnitudes de las fuerzas de interacción. Dado que la mayoría de las poblaciones naturales presentan dinámicas estables (Kendall et al. 1998), aun estando inmersas en comunidades complejas (Dunne \& Pascual 2006); May (1972) impone el desafío de encontrar los mecanismos biológicos actuales que promueven la estabilidad de sistemas de poblaciones que, de considerárseles como entidades físicas en interacción aleatoria, presentarían dinámicas inestables. En este contexto, son variados los mecanismos estabilizadores que se han propuesto en la literatura durante los últimos 37 años (véase McCann 2000). Por elegancia, intuitividad y relevancia teórica, uno de los mecanismos causales de la estabilización poblacional que ha recibido mayor interés entre los ecólogos y evolucionistas es el comportamiento adaptativo de los individuos componentes de las poblaciones (Abrams 2000, Bolker et al. 2003). Entre los distintos tipos de rasgos plásticos adaptativos que se les ha reconocido implicancias importantes para la dinámica y estabilidad de las poblaciones, se encuentran el comportamiento adaptativo de las presas frente a riesgo de depredación (Abrams 1984), la elección de tiempo óptimo de transición de nicho ontogenético (Takimoto 2003), las decisiones de ocupación de microhábitat (Ramos-Jiliberto \& GonzálezOlivares 2000) y la elección óptima de dieta (Krivan 1996, Kondoh 2003). En este trabajo, nuestro propósito es sistematizar y analizar críticamente los avances teóricos que se han desarrollado acerca del rol estabilizador a nivel poblacional, de un tipo de comportamiento adaptativo del cual se posee un mejor entendimiento teórico y soporte empírico a nivel individual: la elección óptima de dieta.

La teoría ecológica de la optimización asume que el comportamiento de los individuos está modulado por la selección natural, maximizando sus adecuaciones biológicas (McArthur \& Pianka 1966). Esta teoría predice que un depredador deberá decidir si captura un determinado ítem presa una vez que lo detecta, o espera a encontrar una presa más redituable (del inglés "profitable"), dependiendo de las abundancias de sus presas (Stephens \& Krebs 1986). En un contexto poblacional dinámico, aunque la calidad de cada individuo presa se asuma constante, la abundancia de presas variará a través del tiempo y afectará directamente las decisiones de forrajeo de sus depredadores, las cuales determinarán a su vez las tasas de cambio en abundancia de ambas poblaciones. De esta forma, la dinámica poblacional afectará las decisiones de forrajeo óptimas de los individuos. El siguiente paso será abordar la relación entre el forrajeo óptimo (FO) de los individuos con la dinámica asintótica (i.e., de largo plazo) de la población que ellos componen, para luego estudiar sus consecuencias a nivel comunitario.

Considerando los efectos del FO sobre la dinámica poblacional, resulta intuitivo esperar que si los individuos de una población son capaces de elegir óptimamente qué presas consumirán en cada instante, en función de sus abundancias y redituabilidad relativa, esta población tendrá una mayor probabilidad de persistir después de una perturbación que afecte la abundancia de sus presas más redituables, comparado a si forrajearan sobre un único conjunto de recursos de manera fija (MacArthur \& Pianka 1966, Kondoh 2003). Por otro lado, el FO controlaría el efecto regulador de la población de depredadores sobre sus presas, debido a que si su población preferida de presas disminuye fuertemente en abundancia, disminuiría su presión de depredación sobre esta al incorporar en su dieta presas menos redituables (Kjellander \& Nordström, 2003). Entonces, tanto para la presa como para el depredador, el FO debiera ser un elemento estabilizador.

En este trabajo, se examina el rol del FO como fuerza estabilizadora de las dinámicas poblacionales. La aproximación de análisis, aquí presentada, es aplicable al estudio del efecto de otros comportamientos adaptativos individuales sobre la establidad poblacional, con la condición que aquellos satisfagan los supuestos de la Teoría Ecológica de la Optimización. En la primera sección se revisan elementos de la Teoría del Forrajeo Óptimo (TFO) que servirán de base para el análisis posterior. En la segunda sección, se examinan trabajos teóricos que han estudiado la relación FO-estabilidad poblacional a niveles crecientes de complejidad estructural: i) cadena trófica, ii) módulo de un depredador y dos presas, iii) módulo con depredación intragremio y iv) 
tramas trófica complejas. En la tercera sección, se analiza esta relación FO-estabilización poblacional en torno a tres preguntas centrales: i) ¿cuál es el sistema control contra el cual se compara la estabilidad de la población cuyos individuos presentan FO?, ii) ¿cuál es el concepto de estabilidad utilizado? y iii) ¿cómo se incorporan los supuestos del FO en las reglas que gobiernan la dinámica poblacional? Posteriormente se presenta una síntesis, especificando puntos cruciales que deben atenderse a fin de evaluar correctamente el rol estabilizador del FO sobre la dinámica poblacional, los cuales debieran también considerarse explícitamente en la evaluación del rol estabilizador de otros comportamientos adaptativos óptimos. Finalmente ofrecemos una nueva dirección de investigación en el ámbito de la relación FOestabilidad poblacional. Aquí, conjeturamos que el efecto estabilizador del FO dependerá cualitativamente tanto del nivel de recursos en el sistema, como de la relación entre tasa de perturbación ambiental y tasa de adaptación del depredador.

\section{ELEMENTOS DE LA TEORÍA DEL FORRAJEO ÓPTIMO}

La elección óptima de dieta es un componente de la teoría del forrajeo óptimo (TFO) y se construye en base a que las presas de un depredador son distintas entre sí, en términos de la energía que le reportan a este y del tiempo que el depredador debe ocupar en la manipulación de cada una de ellas (Stephens \& Krebs 1986). La TFO es un corolario de la teoría de la selección natural (Stephens \& Krebs 1986), en que se maximiza la adecuación biológica durante la actividad de forrajeo. Esta teoría se deriva de un modelo de optimización que se compone de: i) una decisión, i.e., capturar o ignorar una presa una vez encontrada, ii) una moneda de adecuación biológica, i.e., la forma cuantificable en que la decisión tiene consecuencias sobre la adecuación biológica y iii) supuestos restrictivos consistentes con el sistema en estudio (Stephens \& Krebs 1986).

La moneda de adecuación biológica más usada en problemas de elección óptima de dieta es la tasa de ingesta energética a largo plazo
(Stephens \& Krebs 1986). En la TFO, esta tasa se obtiene de la razón entre la ingesta energética total de la actividad de forrajeo $\mathrm{E}$ y el tiempo total que se asignó a esta actividad T. En el caso de un depredador forrajeando sobre dos presas $(\mathrm{i}=1,2)$, la TFO define $\mathrm{E}=\mathrm{T}^{\mathrm{S}}$ $\left(\lambda_{1} \mathrm{E}_{1}+\lambda_{1} \mathrm{E}_{1}\right)$ y $\mathrm{T}=\mathrm{T}^{\mathrm{S}}+\mathrm{T}^{\mathrm{H}}$, donde $\mathrm{T}^{\mathrm{H}}=\mathrm{T}^{\mathrm{S}}\left(\lambda_{1} \mathrm{~h}_{1}+\right.$ $\lambda_{1} h_{1}$ ) es el tiempo de manipulación total y $\lambda_{\mathrm{i}} \mathrm{y} \mathrm{h}_{\mathrm{i}}$ son la tasa de encuentro y tiempo de manipulación, respectivamente. Si la presa $\mathrm{i}=1$ es la más "redituable" (i.e., $\mathrm{E}_{1} \mathrm{~h}_{1}^{-1}>\mathrm{E}_{2} \mathrm{~h}_{2}{ }^{-1}$ ), el depredador la capturará cada vez que la encuentre. El depredador capturará a la presa menos redituable solo si la tasa de ingesta energética a largo plazo (E/T) que le reporta cazar a ambas presas es mayor que cazar solo a la más redituable. Lo anterior se cumple cuando

$$
\frac{1}{\lambda_{1}}<\frac{E_{1}}{E_{2}}\left(h_{1}-h_{2}\right)
$$

condición que no incluye la tasa de encuentro con la presa menos redituable. Entonces, en la TFO se explicita que no basta considerar la redituabilidad relativa de los ítemes presa, sino que también se debe registrar la tasa de encuentro con la presa más redituable, la que, en la mayoría de los casos, depende de su abundancia absoluta (Stephens \& Krebs 1986). Por lo tanto, en un contexto dinámico en que las abundancias de las presas más redituables de un depredador óptimo decrecen bajo el umbral explicitado por la condición (1), este podrá ajustar su comportamiento de forrajeo y ampliar su dieta incorporando presas menos redituables.

El modelo de elección óptima de dieta puede considerarse como el punto de partida para la modelación de estrategias de forrajeo moldeadas por selección natural. Hoy disponemos de avances teóricos que relajan los supuestos de este modelo y permiten considerar comportamientos más realistas que la teoría descrita en los párrafos precedentes. Estos avances incluyen la incorporación de dinámica poblacional, tanto de depredadores como de presas, en el proceso de toma de decisión (Drossel et al. 2001, Kondoh 2003, Uchida et al. 2007), como también la adopción de una aproximación basada en el estado de los individuos (Houston \& McNamara 1999). Las herramientas utilizadas en estos casos son la programación dinámica, teoría de juegos y otras provenientes de la teoría de la 
optimización. El lector interesado puede acudir a las excelentes revisiones hechas por Houston \& McNamara (1999) y Clark \& Mangel (2000). En lo que sigue, nos referiremos por $\mathrm{FO}$ en su sentido amplio, como la acción individual de ajuste de las estrategias de forrajeo conducentes a la maximización del éxito reproductivo.

\section{CONSECUENCIAS POBLACIONALES DEL FORRAJEO ÓPTIMO}

En esta sección se mostrará evidencia teórica del rol estabilizador del FO en sistemas de poblaciones interactuando mediante relaciones tróficas. Los sistemas que se considerarán son: i) cadena trófica, ii) módulo de un depredador y dos presas, iii) módulo con depredación intragremio y iv) tramas trófica complejas.

En sistemas de un depredador óptimo forrajeando sobre una única presa, que es el caso más simple de una cadena trófica, se ha demostrado que la incorporación de este comportamiento adaptativo estabiliza la dinámica de las poblaciones interactuantes (Abrams 1984, Krivan 2007). Dado que el depredador consume una única presa, la decisión que maximice la adecuación biológica ya no será qué presas incluir en su dieta, sino el tiempo que asigna a alimentarse (Abrams 1984) o la inversión en forrajeo (Krivan 2007). $\mathrm{Si}$ el consumidor intermedio en una cadena tritrófica ajusta adaptativamente el tiempo de forrajeo, el sistema presenta un punto de equilibrio interior localmente estable (Abrams 1984), mientras que una cadena tritrófica con comportamiento de forrajeo fijo (i.e., no adaptativo) puede presentar dinámicas inestables, como oscilaciones periódicas e incluso caos determinista (Hasting \& Powell 1991). Por otro lado, en un sistema LotkaVolterra de un depredador y una presa, el ajuste adaptativo de la actividad de forrajeo por parte del depredador impone un límite inferior a las oscilaciones típicas de este sistema (Krivan 2007). Así el forrajeo adaptativo impide que los tamaños poblacionales caigan bajo valores críticos en que la estocasticidad pueda conducir a las poblaciones interactuantes a la extinción.

En sistemas de un depredador forrajeando sobre dos presas que le reportan distinto rendimiento de forrajeo, la teoría de la elección óptima de dieta es directamente aplicable mediante la utilización de la respuesta funcional tipo II, para cada presa (Holt \& Kimbrell 2007). En estos sistemas, la incorporación de forrajeo adaptativo puede permitir su permanencia, es decir, la coexistencia indefinida de las tres poblaciones interactuantes (Krivan \& Sikder 1999). Además, comparado con un sistema en que el depredador tiene un comportamiento de forrajeo fijo sobre ambas presas, la incorporación del forrajeo adaptativo reduce la amplitud de las oscilaciones poblacionales (Krivan 1996). Sin embargo, si se compara con un sistema en que el depredador se especializa en la presa que le brinda un mayor rendimiento de forrajeo, la región de parámetros que permite la permanencia es menor cuando el forrajeador posee comportamiento adaptativo (Krivan \& Sikder 1999)

También se ha demostrado que el forrajeo adaptativo estabiliza los sistemas tritróficos que exhiben depredación intragremio (aquellos en que el depredador compite con su presa por un recurso compartido) (Okuyama \& Ruyle 2003, Krivan \& Diehl 2005). Holt \& Polis (1996) muestran que estos sistemas son inestables a niveles de productividad muy alta o muy baja del recurso, siendo la coexistencia de las tres especies posible solo a niveles intermedios de productividad del recurso. Tanto cuando es el consumidor intermedio el que forrajea óptimamente (Okuyama \& Ruyle 2003), como cuando es su depredador (Krivan \& Diehl 2005), las regiones de coexistencia se amplían, comparado con un escenario en que consumidor y depredador mantienen fijo su comportamiento de forrajeo.

Finalmente, la incorporación de $\mathrm{FO}$ en poblaciones interactuando en una trama trófica compleja, estabiliza la dinámica de esta red ecológica, incrementando la persistencia de las especies que la componen (Brose et al. 2003, Kondoh 2003, 2006, Uchida et al. 2007). Más aún, cuando una alta proporción de las especies que componen la red son forrajeadores óptimos y presentan una respuesta rápida a cambios en la abundancia de sus presas más redituables, la relación negativa de complejidad-estabilidad que encuentra May (1972) llega a invertirse 
(Kondoh 2003). Sin embargo, Brose et al. (2003) discuten esta conclusión, argumentando que los modelos de dinámica poblacional y de ensamblamiento de red utilizados por Kondoh (2003) son inapropiados. Utilizando modelos diferentes, Brose et al. (2003) encuentran que, aunque el FO estabiliza la dinámica de las redes estudiadas, no es capaz de revertir la relación negativa complejidad-estabilidad. Para resolver el debate, Kondoh (2006) identifica que los procedimientos de Brose et al. (2003) generan una disminución en el número de especies basales, lo cual desestabilizaría las tramas tróficas y explicaría la discrepancia. De este debate, se extrae que el efecto estabilizador del FO podría ser dependiente de la estructura de la red estudiada.

\section{ANÁLISIS DEL ROL ESTABILIZADOR DEL FO}

Los trabajos que se discutieron en la sección previa, apoyan la hipótesis de que el FO estabiliza los sistemas de poblaciones que interactúan mediante relaciones tróficas. Sin embargo, una evaluación más profunda de esta hipótesis requiere necesariamente precisar ciertos criterios de análisis, que nosotros trasladamos a tres preguntas fundamentales: ¿con respecto a qué sistema es comparable la estabilidad de un modelo que incorpora forrajeo adaptativo?, ¿qué concepto de estabilidad se utiliza?, y ¿cómo se modela e incorpora el comportamiento óptimo de un depredador en su dinámica poblacional? A continuación se desarrollan estos tres puntos.

\section{i) ¿Con respecto a qué sistema es comparable la estabilidad de un modelo que incorpora forrajeo adaptativo?}

Para probar si el FO estabiliza la dinámica de sistemas de poblaciones interactuando mediante relaciones tróficas, se debe explicitar el sistema basal que llega a ser estabilizado y controlar los factores distintos al FO que podrían afectar su estabilidad. En este sentido, la dinámica de un sistema que incorpora forrajeo adaptativo se debe contrastar con la dinámica de un sistema que posea el mismo número de especies interactuantes, el mismo número de interacciones y el mismo esfuerzo de forrajeo promedio que el depredador asigna a sus presas, pero en que el comportamiento de forrajeo permanezca constante en el tiempo. En la literatura existen estudios en los que se compara la dinámica de sistemas que incorporan forrajeo adaptativo en el depredador con la dinámica de sistemas en que el depredador no adaptativo es generalista (Krivan 1996, Krivan \& Sikder 1999, Okuyama \& Ruyle 2003, Kondoh 2003, Krivan \& Diehl 2005) y también en los que se compara con respecto a la dinámica de sistemas en que el depredador es especialista sobre la presa más redituable (Krivan 1996, Krivan \& Sikder 1999). Sin embargo, ambas comparaciones no son controles válidos del efecto del forrajeo adaptativo sobre la estabilidad de estos sistemas. Por un lado, la comparación con la estrategia especialista varía el número de interacciones, por lo que no se despeja el efecto negativo que la complejidad ejerce sobre la estabilidad del sistema (May 1972). La comparación con la estrategia generalista no controla por la fuerza de interacción, suponiendo además que el depredador asigna el mismo esfuerzo de forrajeo sobre ambas presas. Aquí enfatizamos que la característica del forrajeador óptimo, que lo diferencia del especialista y del generalista (no adaptativos), es su capacidad para adaptar su esfuerzo de forrajeo según la abundancia de sus presas más redituables. Este es capaz de asignar esfuerzo en forrajear a presas menos redituables cuando la abundancia de las de mayor redituabilidad bajan de cierto umbral. Entonces, la comparación con un depredador generalista debiera hacerse conservando el esfuerzo diferencial asignado a cada presa, en que solo se diferencie que el forrajeador óptimo varía este esfuerzo diferencial según la abundancia de las presas más redituables, mientras que el generalista los mantiene fijos. Es así como la utilización de un "contraste topológico”, es decir, la comparación entre sistemas en base al número de especies o de interacciones, es insatisfactorio como control del comportamiento adaptativo del depredador, pues no controla adecuadamente la dimensionalidad del sistema (RamosJiliberto et al. 2004) ni las magnitudes de interacción (Fig. 1). En este trabajo, se propone el "contraste estructural" para evaluar las consecuencias del comportamiento 
adaptativo del depredador. Esto es, la comparación de sistemas que comparten la misma topología y fuerza de interacción relativa promedio, en el que varíe solo la capacidad (sí/no) del depredador de adaptar el esfuerzo de forrajeo relativo entre sus presas. En la Fig. 1 se representan el contraste topológico, usualmente utilizado en la literatura, y el contraste estructural propuesto. Para efectuar el contraste estructural de forma numérica, se deben ejecutar un número de realizaciones del modelo con comportamiento adaptativo y promediar los esfuerzos de forrajeo relativos. Estos promedios podrían utilizarse como los valores de los esfuerzos relativos que permanecerán fijos en el sistema control, no adaptativo (Fig. 1).

\section{ii) ¿De acuerdo a qué concepto de estabilidad el FO estabiliza?}

Son variados los conceptos de estabilidad que se han utilizado en ecología (ver McCann 2000). En el caso del análisis de las consecuencias dinámicas del $\mathrm{FO}$, se ha utilizado el concepto de estabilidad local (Abrams 1984, Krivan 1996, Okuyama \& Ruyle 2003) y los conceptos de permanencia (Krivan

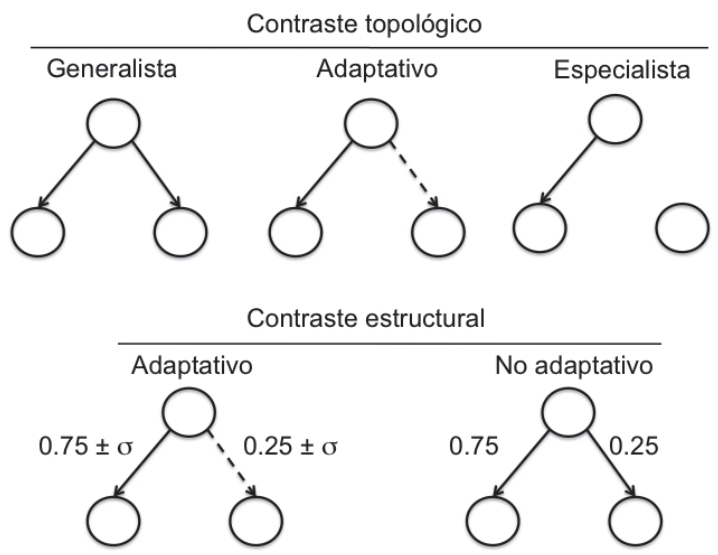

Fig. 1: Dos tipos de contraste para evaluar el rol estabilizador del forrajeo óptimo en sistemas de poblaciones interactuando mediante relaciones tróficas. En números se indica el promedio del esfuerzo de forrajeo sobre varias realizaciones para un depredador adaptativo, siendo o la desviación estándar.

Two kinds of contrast for evaluating the stabilization role of optimal foraging on population systems. Numbers indicate the average foraging efforts over various realizations for an optimal forager, being $\sigma$ the standard deviation over these realizations.
\& Sikder 1999, Krivan \& Diehl 2005, Uchida et al. 2007) y persistencia (Kondoh 2003, Brose et al. 2003). El concepto de estabilidad local se refiere a la existencia de un punto de equilibrio localmente estable, es decir, a la existencia de valores de tamaños poblacionales invariantes en el tiempo (Fig. 2) y que tras una pequeña perturbación, las poblaciones retornan a estos mismos valores (May 1972). Los conceptos de permanencia y persistencia no descansan en la existencia de un punto de equilibrio localmente estable, sino en si las especies perduran o no en el sistema. Técnicamente, se dice que un sistema es permanente si la frontera del cuadrante positivo es un repulsor (Krivan \& Sikder 1999), es decir, la permanencia de un sistema se define por la existencia de un límite inferior positivo para las densidades poblacionales (Fig. 2). En cambio, la persistencia se determina usualmente mediante simulación numérica de las trayectorias poblacionales, y se define como la cantidad de poblaciones iniciales del sistema cuyo tamaño supera cierto umbral de extinción, después de un número determinado de iteraciones del modelo (Fig. 2) (e.g., Valdovinos et al. 2009).

El concepto de estabilidad que se utilice dependerá de la pregunta que se quiera responder, pues las consecuencias que se desprendan del análisis estarán estrechamente ligadas al concepto utilizado. Si la pregunta se relaciona con la conservación de una o más especies interactuantes, es más adecuado la utilización del concepto de persistencia, pues la no existencia de un punto de equilibrio localmente estable no implica necesariamente que la población tenga un mayor riesgo de extinción (Krivan 2007). Además, mientras mayor la riqueza específica del sistema, más difícil es definir las condiciones de existencia y estabilidad de los equilibrios. De esta manera se explica el hecho que los trabajos en tramas tróficas complejas que incorporan FO, ocupen los conceptos de persistencia (Brose et al. 2003, Kondoh 2003) y permanencia (Uchida et al. 2007).

iii) ¿Cómo se incorpora el FO en la dinámica poblacional del sistema que estabiliza?

Un problema de optimización consiste en encontrar los máximos (o mínimos) globales 
de una función objetivo sujeta a un conjunto de restricciones. La TFO postula que un organismo se comporta maximizando su éxito reproductivo, por lo que la función objetivo que describe el comportamiento de un forrajeador óptimo es alguna moneda de su adecuación biológica (Stephens \& Krebs 1986). Los máximos globales de la función objetivo se obtienen al optimizar ciertas variables de decisión, es decir, encontrando la decisión óptima del individuo (Stephens \& Krebs 1986). Las variables de decisión en el ámbito conductual son aquellas variables que el organismo es capaz de controlar, como son la cantidad de energía o tiempo que destina a una cierta actividad. Entonces, una decisión óptima es el conjunto de valores de cada una de estas variables que determinan un máximo global de la función objetivo (Stephens \& Krebs 1986).

En modelos poblacionales que incorporan supuestos adaptativos, la moneda de adecuación biológica que se ha utilizado como función objetivo es la tasa instantánea de crecimiento per capita (Abrams 1984, Krivan
1996, Krivan \& Sikder 1999, Brose et al. 2003, Kondoh 2003, Okuyama \& Ruyle 2003, Krivan $\&$ Diehl 2005), la que puede formalizarse como $\mathrm{dN}_{\mathrm{i}} \mathrm{N}_{\mathrm{i}} \mathrm{dt}^{-1}=\mathrm{n}_{\mathrm{i}}-\mathrm{m}_{\mathrm{i}}$, donde $\mathrm{n}_{\mathrm{i}} \mathrm{y} \mathrm{m}_{\mathrm{i}}$ son las tasas de natalidad y mortalidad per cápita de la población de forrajeadores $\mathrm{i}$ de tamaño $\mathrm{N}_{\mathrm{i}}$, respectivamente. La variable de decisión que ha sido usada en estos modelos es el esfuerzo de forrajeo que asigna la población de forrajeadores $\mathrm{i}$ a la población de presas $\mathrm{j}, \mathrm{a}_{\mathrm{ij}}$ (Kondoh 2003, Okuyama \& Ruyle 2003). El esfuerzo de forrajeo $a_{i j}$ puede considerarse como actividad de forrajeo (Krivan 2007), como tiempo destinado a esta actividad (Abrams 1984) o también como probabilidad de ataque una vez encontrada la presa (Krivan 1996, Krivan \& Sikder 1999, Krivan \& Diehl 2005). Entonces, la dinámica de una población de forrajeadores óptimos i que es depredada por una población $\mathrm{k}$ y que consume a la población de recursos $\mathrm{j}$, puede describirse como

$$
\frac{d N_{i}}{N_{i} d t}=n_{i}\left(N_{i}, N_{j}, a_{i j}\right)-m_{i}\left(N_{i}, N_{k}, a_{i j}\right)
$$

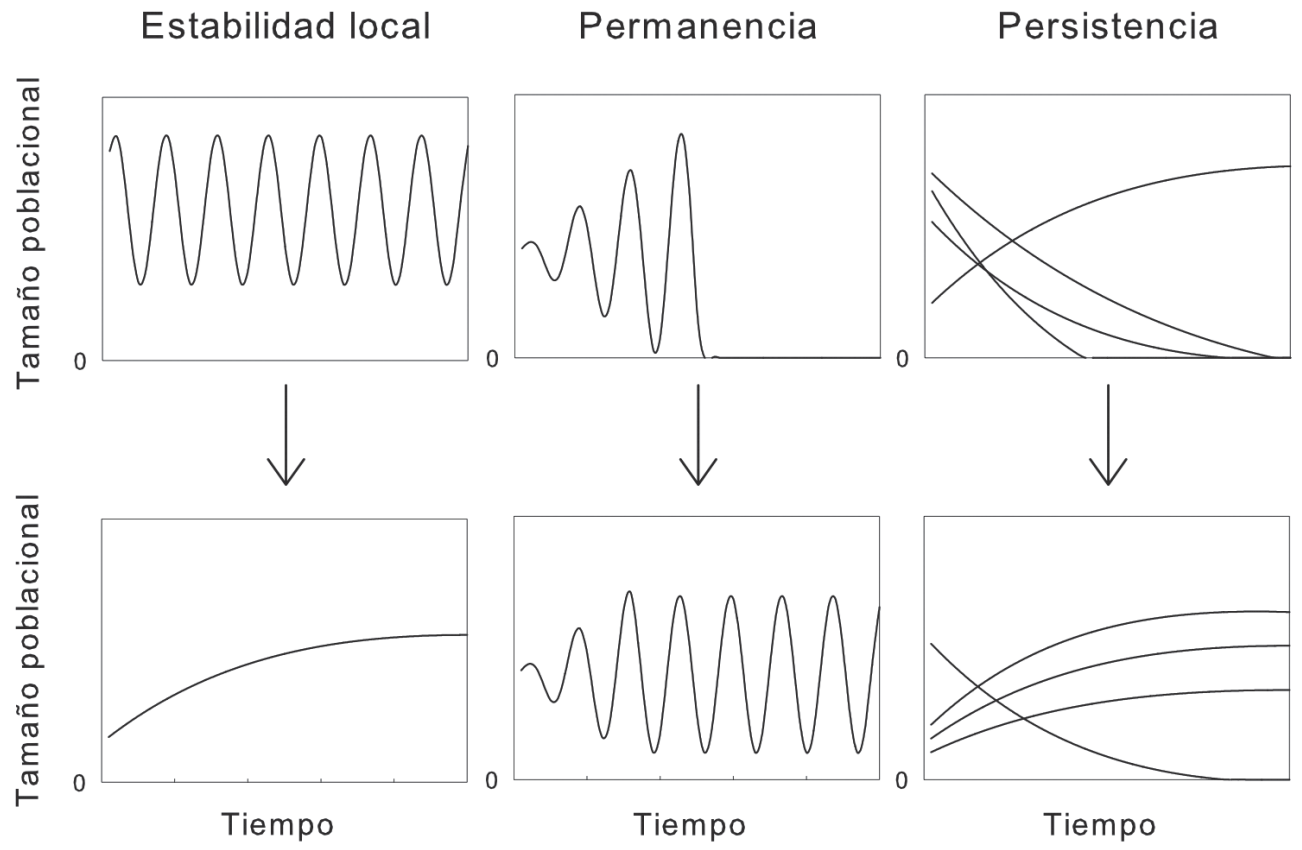

Fig. 2: Estabilización de dinámicas poblacionales, de acuerdo a tres conceptos de estabilidad utilizados en estudios teóricos acerca del rol estabilizador del forrajeo óptimo. Las flechas indican aumento de estabilidad, de acuerdo a cada concepto.

Stabilization of population dynamics according to three stability concepts used in theoretical studies about the stabilizing role of optimal foraging. Arrows indicate increase in stability. 
donde la solución del problema de optimización consiste en encontrar $\mathrm{a}_{\mathrm{ij}}$ que cumpla con

$\frac{\partial}{\partial a_{i j}}\left(\frac{d N_{i}}{N_{i} d t}\right)=0$, dado $\frac{\partial}{\partial^{2} a_{i j}}\left(\frac{d N_{i}}{N_{i} d t}\right)^{2}<0$

(Houston \& McNamara 1999).

Por simplicidad, en buena parte de la literatura se asume que el depredador ajusta sus esfuerzos de forrajeo de manera instantánea, según varíen las abundancias de sus presas más redituables, lo que corresponde a encontrar $\operatorname{los} \mathrm{a}_{\mathrm{ij}}$ sujetos a la condición (3) en cada iteración del modelo y evaluarlos en la ecuación (2) (Abrams 1984, 1992). Sin embargo, revisiones sobre estudios empíricos de forrajeo han concluido que este esfuerzo no se ajusta de manera instantánea (véase Stephens \& Krebs 1986). En consecuencia, se requieren modelos que incluyan ecuaciones que describan tasas de cambio de los esfuerzos de forrajeo, según la disponibilidad de recursos para el depredador (Abrams 1992, Kondoh 2003, Uchida et al. 2007). Para esto, se define $\mathrm{a}_{\mathrm{ij}}$ como una variable de estado adicional, cuya ecuación que gobierna la tasa de cambio de los esfuerzos de forrajeo que el depredador asigna a cada presa es

$$
\frac{d a_{i j}}{a_{i j} d t}=G_{i}\left[e_{i j} f_{i j}\left(N_{j}\right)-\sum_{k \in \text { presas }} e_{i k} f_{i k}\left(N_{k}\right)\right]
$$

(Kondoh 2003, Uchida et al. 2007). En (4), $\mathrm{f}_{\mathrm{ij}} \mathrm{y}$ $\mathrm{e}_{\mathrm{ij}}$ son la respuesta funcional y una medida de la eficiencia de conversión a crecimiento poblacional de la especie i para cada una de sus presas j respectivamente, mientras $G_{i}$ es la tasa de adaptación del depredador i (Kondoh 2003, Uchida et al. 2007). Esta tasa de cambio conserva la concepción de optimización, pues define que el esfuerzo de forrajeo sobre la presa $\mathrm{j}$ incrementa en el tiempo mientras mayor sea la cantidad de energía o biomasa que esta le reporte, en relación a la que obtenga del resto de sus presas. La ecuación (4) impone un retardo a la toma de la decisión óptima de los forrajeadores, pues la tasa de cambio explicita que existe un proceso de ejecución de la decisión que demora un cierto tiempo (Abrams 1992). Cabe notar que se puede derivar una respuesta instantánea desde la ecuación (4), si es que se asume que la tasa de ajuste $G_{i}$ es infinita. Sin embargo, aún no existen suficientes resultados teóricos que permitan llegar a un consenso acerca del efecto diferencial del ajuste instantáneo versus retardado de los $\mathrm{a}_{\mathrm{ij}}$. Abrams (1992) encuentra que el efecto cualitativo del FO sobre la dinámica de un sistema de un depredador forrajeando sobre una única presa, es independiente de la velocidad de ajuste del $\mathrm{a}_{\mathrm{ij}}$. Sin embargo, Kondoh (2003) evidencia que la relación complejidad-estabilidad en tramas tróficas complejas (sensu May 1972) se hace positiva cuando se incorpora $F O$ con alta $G_{i}$, mientras que a bajos $G_{i}$ la relación permanece negativa (Kondoh 2003). Estos resultados divergentes indican, por lo tanto, que este tópico requiere resolución futura.

En los párrafos precedentes se ha revisado la función objetivo y la variable de decisión que han sido utilizadas en modelos poblacionales que incorporan FO. En lo que sigue se describirán los tipos de restricciones que se han incorporado en estos modelos, eligiéndose aquellas que pueden generalizarse a cualquier comportamiento adaptativo óptimo. Estas son: i) el compromiso entre la ganancia, en términos de natalidad, que le reporta a los organismos de una población una cierta conducta, así como sus costos en términos de mortalidad (Lima \& Dill 1990) y ii) restricciones entre las opciones de decisión que enfrenta el organismo (Murdoch et al. 1975). En cuanto al FO, el compromiso explicitado en i) se expresa entre la ganancia de un mayor esfuerzo de forrajeo sobre los recursos y los costos de este esfuerzo en términos de mortalidad por depredación (Lima \& Dill 1990). Bajo esta restricción, los organismos se comportan maximizando su moneda de fitness, al encontrar un valor óptimo de $\mathrm{a}_{\mathrm{ij}}$ que responda al compromiso entre $\mathrm{n}_{\mathrm{i}}\left(\mathrm{a}_{\mathrm{ij}}\right)$ y $\mathrm{m}_{\mathrm{i}}\left(\mathrm{a}_{\mathrm{ij}}\right)$ (Abrams 1984, Krivan 2007). Cabe hacer notar que el depredador solo forrajeará sobre su presa $\mathrm{j}$ si $\mathrm{n}_{\mathrm{i}}\left(\mathrm{a}_{\mathrm{ij}}\right)$ > $\mathrm{m}_{\mathrm{i}}\left(\mathrm{a}_{\mathrm{ij}}\right)$, es decir, cuando los beneficios de esta actividad sean mayores a sus costos (Krivan 2007). La restricción explicitada en ii) emerge cuando el depredador forrajea sobre dos o más presas (i.e., $\mathrm{j}=1,2, \ldots \mathrm{n}$ ), pues la decisión óptima del forrajeador consistirá en un conjunto de $\mathrm{a}_{\mathrm{ij}}$ que determinarán a qué presas atacará y en qué magnitud relativa (Krivan 1996, Krivan \& Sikder 1999, Kondoh 2003). En este contexto, la maximización del éxito 
reproductivo se reduce a la maximización de la tasa de ingesta energética mientras forrajea, en el mismo sentido que en la elección óptima de dieta (Stephens \& Krebs 1986), descrita al comienzo de este trabajo. Entonces, el depredador debe asignar una cantidad total fija de esfuerzo a la actividad de forrajeo, la cual se reparte entre los distintos $\mathrm{a}_{\mathrm{ij}}$; esto es $\sum_{j} a_{i j}=1$ (e.g., Kondoh 2003). Con esta forma lineal de la restricción entre las opciones del forrajeador, se asume que no existe interacción entre los esfuerzos de forrajeo que asigna el depredador sobre sus distintas presas, es decir, la asignación de esfuerzo hacia una presa determinada no incrementa el éxito de captura de otra presa (Uchida et al. 2007). Sin embargo, esta independencia entre los esfuerzos de forrajeo del depredador es solo esperable cuando sus presas se encuentran en diferentes subhábitats (Murdoch et al. 1975). En hábitats relativamente homogéneos o con pocas subdivisiones, los depredadores encuentran también a sus presas alternativas cuando buscan a las más redituables (Murdoch et al. 1975); entonces el esfuerzo de forrajeo asignado a una presa particular incrementaría el esfuerzo asignado a una presa alternativa. Cuando el esfuerzo de forrajeo sobre una presa aumenta o disminuye el esfuerzo de forrajeo ejercido sobre otra presa, las restricciones sobre los esfuerzos de forrajeo son no lineales (Uchida et al. 2007), lo que puede expresarse como

$$
\sum_{j} a_{i j}{ }^{x}=1
$$

donde el exponente $\mathrm{x}$ indica la magnitud $\mathrm{y}$ signo de la interacción entre los esfuerzos de forrajeo (Uchida et al. 2007). Esta es un área de muy reciente desarrollo y las consecuencias de incorporar el supuesto realista, representado por restricciones no lineales, es materia de investigación actual y futura.

\section{SÍNTESIS Y PERSPECTIVAS FUTURAS}

En el presente trabajo, se analizaron algunas de las consecuencias que un rasgo, a nivel de organismos, tiene sobre la estabilidad de las poblaciones que estos componen.
Específicamente, se consideró la conducta de forrajeo de los individuos y se revisó parte de una teoría que se ha desarrollado para explicarla, la TFO (Stephens \& Krebs 1986), en el contexto relevante para la dinámica de poblaciones. En base a lo que se expuso acerca de la TFO en la primera sección, en la sección dos mostramos que existe consistencia en la literatura en torno al rol estabilizador del FO sobre las dinámicas poblacionales (e.g., Abrams 1984, Kondoh 2003, Krivan \& Diehl 2005). Sin embargo, en la sección tres mostramos que la hipótesis de estabilización por FO ha sido sometida a pruebas teóricas débiles, lo que revela ciertos vacíos analíticos que pueden resultar claves para estudios similares de casos más controversiales o de menor desarrollo que el FO. La falta de un sistema control adecuado y la diversidad o ambigüedad del concepto de estabilidad utilizado, son aspectos que deben considerase rigurosamente en trabajos futuros. Otro aspecto que debe controlarse en el análisis de respuestas adaptativas, es la homogeneidad de supuestos fundamentales. En el caso del FO, parece haber congruencia en relación a la función objetivo (e.g., Abrams 1984, Krivan 1996, Okuyama \& Ruyle 2003) y a las variables de decisión adecuada (e.g., Abrams 1992, Kondoh 2003, Uchida et al. 2007). Sin embargo, aún queda por resolver el rol de la dinámica de la variable de decisión (instantánea o retardada) (Abrams 1992, Kondoh 2003) sobre la relación estabilización poblacional-FO. Un último aspecto a considerar, son las restricciones que solo recientemente han permitido incorporar no linealidad (Uchida et al. 2007), aun siendo este supuesto el que mejor representa el fenómeno natural (Murdoch et al. 1975).

Tras el análisis crítico realizado en este trabajo, proponemos que los puntos que se deben considerar, a fin de evaluar correctamente el rol estabilizador de cualquier comportamiento adaptativo óptimo sobre la dinámica poblacional, son: i) la definición de un sistema control cuya respuesta sea comparable con la de un modelo que incorpora comportamiento adaptativo, ii) la delimitación explícita del concepto de estabilidad a utilizar y iii) la explicitación de los componentes del problema de optimización que se usarán en el modelo (i.e., función objetivo, variables de 
decisión y restricciones). Aquí se presenta el contraste estructural como procedimiento que permite controlar correctamente las consecuencias de comportamientos adaptativos sobre la estabilidad poblacional. Este contraste se plantea como la comparación del modelo que incorpora comportamiento adaptativo, con un sistema que comparta la misma topología de opciones para la variable de decisión y la misma probabilidad de elección relativa promedio asignada a cada opción (Fig. 1). Como ya se discutió en la sección tres, las consecuencias de la dinámica de las variables de decisión sobre la dinámica poblacional, es aún materia de investigación. El tiempo que le toma a los organismos ejecutar una cierta decisión, puede ser especialmente relevante cuando se consideran respuestas fenotípicas adaptativas como el desarrollo de defensas morfológicas o cambios en el uso de hábitat (Garay-Narváez \& RamosJiliberto 2009). En cuanto a las restricciones que estarían ejerciendo control sobre un comportamiento óptimo, es importante considerar aquellas que denotan interacción entre las opciones que se le presentan al individuo (e.g., Murdoch et al. 1975), pues las consecuencias poblacionales de esta no linealidad pueden ser fuertes. Retardo en la expresión de opciones conductuales y estructura de las restricciones, constituyen tópicos relevantes y de interesante proyección para la investigación futura en este campo.

Dado el efecto directo que tiene la ingesta sobre el éxito reproductivo de un organismo, puede considerarse biológicamente razonable asumir que el comportamiento de forrajeo de los individuos está moldeado por selección natural, y que estos individuos, por tanto, pueden comportarse como forrajeadores óptimos, maximizando así su adecuación biológica (Stephens \& Krebs 1986). Sin embargo, son comparativamente pocos los modelos poblacionales que incorporan explícitamente el comportamiento de forrajeo adaptativo. La mayoría de los autores utilizan modelos poblacionales en los que se asume que los individuos se comportan como partículas incapaces de ajustar su fenotipo a la variación ambiental (ver Ramos-Jiliberto 2005). La relación negativa entre complejidad y estabilidad que encuentra May (1972) se obtiene como resultado de un sistema de ecuaciones que representan las tasas de cambio demográfico de un conjunto de poblaciones interactuando al azar. No obstante, tal como se hizo énfasis en este trabajo, los individuos también tienen sus reglas dinámicas referentes a las decisiones conductuales que van tomando durante su ontogenia (Stephens \& Krebs 1986). Entonces, además de los mecanismos estabilizadores relacionados con la estructura de las interacciones y los procesos de retroalimentación que de ellas se deriva (McCann et al. 1998), existe evidencia teórica que apoya que el comportamiento adaptativo de los individuos es un buen candidato de solución de la paradoja de May (Kondoh 2003). Un camino abierto para investigación futura es analizar la interacción entre el FO y diversos factores que, por separado, han demostrado actuar como modificadores de la relación negativa encontrada por May (1972). Estos incluyen incidencia de interacciones débiles (McCann et al. 1998), distribución de tamaños corporales entre depredadores y presas (Brose et al. 2006, Otto et al. 2007), omnivoría (Fagan 1997, McCann \& Hastings 1997) y compartimentalización (Pimm \& Lawton 1980, Melián \& Bascompte 2002).

En el actual contexto global en que la biodiversidad es amenazada por múltiles causas asociadas a la expansión demográfica humana (Sisk et al. 1994), cobra especial interés el estudio de los efectos de perturbaciones ambientales sobre la estructura y función de las comunidades. Sin embargo, a nuestro conocimiento existe escaso o nulo desarrollo teórico respecto a las implicancias comunitarias del FO en un contexto de perturbaciones, por ejemplo la pérdida acelerada de especies o bajas drásticas de sus abundancias. ¿Qué estrategia de forrajeo brindaría a la comunidad mayor tolerancia al ataque sobre algunos de sus componentes? Nosotros conjeturamos que si la tasa de adaptación de los forrajeadores óptimos es baja, en relación a la tasa a la que ocurren las perturbaciones, estos tendrían una menor probabilidad de sobrevivir y reproducirse que los generalistas no adaptativos equivalentes (Fig. 3). Esto ocurriría debido a que los forrajeadores óptimos que estén consumiendo a sus presas más redituables, no tendrían la capacidad de ajustar oportunamente su 
esfuerzo de forrajeo si es que estas son suprimidas por la perturbación. Por otro lado, un generalista no adaptativo mantiene una redundancia de interacciones que le permite tolerar la eliminación de algunos de sus ítemes presa, otorgando robustez a su comunidad frente a eventuales pérdidas de componentes (Dunne et al. 2002). Sin embargo, el argumento anterior es esperable solo a niveles suficientemente elevados de recursos basales. Nosotros anticipamos que a niveles bajos de recursos, la estrategia de generalismo fijo sería favorecida (Fig. 3), pues con bajas abundancias de presas el forrajeador óptimo se comportará de la misma forma que el generalista fijo, pero conservando los costos de la plasticidad. En consecuencia, bajo nuestra conjetura la relación directa entre estabilidad poblacional y FO aplicaría solo bajo una combinación particular de niveles tanto de recursos como de magnitud relativa de las tasas de perturbación y adaptación (Fig. 3).

A pesar de los notables avances teóricos logrados hasta hoy en ecología, estamos aún en una etapa temprana de desarrollo de la investigación en sistemas dinámicos multiespecíficos. En los años que siguen, se espera que nuestro entendimiento de los

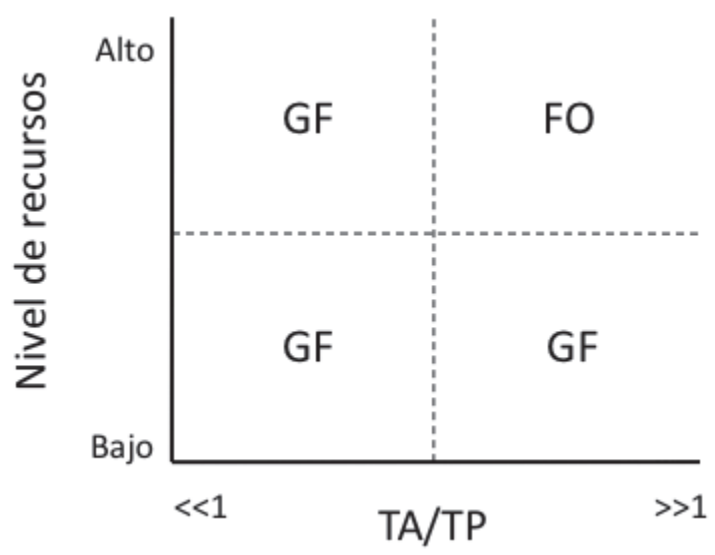

Fig. 3: Estrategia de forrajeo (forrajeo óptimo FO, generalismo fijo GF) que se espera sea seleccionada según nivel de recursos y relación entre tasa de adaptación del forrajeador óptimo y tasa de perturbaciones. TA y TP, refieren a tasa de adaptación del forrajeador óptimo (TA) y tasa de perturbación (TP).

Foraging strategy (optimal foraging FO, fixed generalism GF) expected to be selected, as a function of resource level and the ratio of adaptation rate (TA) to perturbation rate (TP). sistemas naturales progrese cualitativamente a través de un programa de investigación teórica capaz de integrar apropiadamente las restricciones dinámicas relacionadas con la dimensión del sistema y la densidad de interacciones, las restricciones biológicas a la estructura de las comunidades, así como la dinámica acoplada de organismos, poblaciones $\mathrm{y}$ factores ambientales.

\section{AGRADECIMIENTOS}

Este trabajo fue financiado por proyecto FONDECYT 1090132. F. S. Valdovinos agradece el apoyo otorgado por CONICYT a través de una beca de doctorado. Los autores agradecen también a R. O. Bustamante y R. A. Vásquez por su participación en enriquecedoras discusiones que facilitaron la realización de este trabajo.

\section{LITERATURA CITADA}

ABRAMS PA (1984) Foraging time optimization and interactions in food webs. The American Naturalist 124: 80-96.

ABRAMS PA (1992) Adaptive foraging by predators as a cause of predator-prey cycles. The American Naturalist 6: 56-72.

ABRAMS PA (2000) The evolution of predator-prey interactions: Theory and evidence. Annual Review of Ecology and Systematics 31: 79-105.

BOLKER B, M HOLYOAK, V KRIVAN, L ROWE \& O SCHMITZ (2003) Connecting theoretical and empirical studies of trait-mediated interactions. Ecology 84: 1101-1114.

BROSE U, RJ WILLIAMS \& ND MARTÍNEZ (2003) Comment on "Foraging adaptation and the relationship between food-web complexity and stability." Science 301: 918b-918c.

BROSE U, RJ WILLIAMS \& ND MARTÍNEZ (2006) Allometric scaling enhances stability in complex food webs. Ecology Letters 9: 1228-1236.

CLARK CW \& M MANGEL (2000) Dynamic state variable models in ecology: Methods and applications. Oxford University Press, New York, New York.

DUNNE JA, RJ WILLIAMS \& ND MARTÍNEZ (2002) Food-web structure and network theory: The role of connectance and size. Proceedings of the National Academy of Sciences USA 99: 1291712922.

GARAY-NARVÁEZ L \& R RAMOS-JILIBERTO (2009) Induced defenses within food webs: The role of community trade-offs, delayed responses, and defense specificity. Ecological Complexity 6: 383-391.

HASTINGS A \& T POWELL (1991) Chaos in a threespecies food chain. Ecology 72: 896-903.

HOLT D \& T KIMBRELL (2007) Foraging and population dynamics. En: Stephens DW, JS Brown \& RC Ydenberg (eds) Foraging behavior and ecology: 365-396. The University Chicago Press, Chicago, Londres. 
HOLT RD \& GA POLIS (1997) A theoretical framework for intraguild predation. The American Naturalist 149: 745-764.

HOUSTON AI \& JM McNAMARA (1999) Models of adaptive behaviour: An approach based on state. Cambridge University Press, New York.

HUTCHINSON GE (1959) Homage to Santa Rosalia or why are there so many kinds of animals? The American Naturalist 93: 145-159.

KENDALL BE, J PRENDERGAST \& ON BJØRNSTAD (1998) The macroecology of population dynamics: Taxonomic and biogeographic patterns in population cycles. Ecology Letters 1: 160-164.

KONDOH M (2003) Foraging adaptation and the relationship between food-web complexity and stability. Science 299: 1388-1391.

KONDOH M (2006) Does foraging adaptation create the positive complexity-stability relationship in realistic food-web structure? Journal of Theoretical Biology 238: 646-651.

KRIVAN V (1996) Optimal foraging and predator-prey dynamics. Theoretical Population Biology 49: 265-290.

KRIVAN V (2007) The Lotka-Volterra predator-prey model with foraging-predation risk trade-offs. The American Naturalist 170: 771-782.

KRIVAN V \& S DIEHL (2005) Adaptive omnivory and species coexistence in tri-trophic food webs. Theoretical Population Biology 67: 85-99.

KRIVAN V \& A SIKDER (1999) Optimal foraging and predator-prey dynamics II. Theoretical Population Biology 55: 111-126.

KJELLANDER P \& J NORDSTROM (2003) Cyclic voles, prey switching in red fox, and roe deer dynamics - a test of the alternative prey hypothesis. Oikos 101: 338-344.

LIMA SL \& LM DILL (1990) Behavioural decisions made under the risk of predation: A review and prospectus. Canadian Journal of Zoology 68: 61940.

MAY RM (1972) Will a large complex system be stable? Nature 238: 413-414.

McARTHUR RH (1955) Fluctuations of animal populations and a measure of community stability. Ecology 36: 533-536.

McARTHUR RH \& ER PIANKA (1966) Optimal use of a patchy environment. The American Naturalist 100: 603-609.

McCANN KS (2000) The diversity-stability debate. Nature 405: 228-233.
McCANN KS \& A HASTINGS (1997) Re-evaluating the omnivory-stability relationship in food webs. Proceedings of the Royal Society B 264: 12491254.

McCANN K, A HASTINGS \& GR HUXEL (1998) Weak trophic interactions and the balance of nature. Nature 395: 794-798.

MELIÁN CJ \& J BASCOMPTE (2002) Complex networks: Two ways to be robust? Ecology Letters 5: 705-708.

MURDOCH WW, S AVERY \& MEB SMITH (1975) Switching in predatory fish. Ecology 56: 10941105.

OKUYAMA T \& RL RUYLE (2003) Analysis of adaptive foraging in an intraguild predation system. Web Ecology 4: 1-6.

OTTO SB, BC RALL \& U BROSE (2007) Allometric degree distributions facilitate food-web stability. Nature 450: 1226-1230.

PASCUAL M \& JA DUNNE (2006) Ecological networks: Linking structure to dynamics in food webs. Oxford University Press, New York.

PIMM SL \& JH LAWTON (1980) Are food webs divided into compartiments? Journal of Animal Ecology 49: 879-898.

RAMOS-JILIBERTO R (2005) Resource-consumer models and the biomass conversion principle. Environmental Modelling \& Software 20: 85-91.

RAMOS-JILIBERTO R \& E GONZÁLEZ-OLIVARES (2000) Relating behavior to population dynamics: A predator-prey metaphysiological model emphasizing zooplankton diel vertical migration as an inducible response. Ecological Modeling 127: 221-233.

RAMOS-JILIBERTO R, F HOECKER-ESCUTI \& J MENA-LORCA (2004) Why the dimension matters in ecological models? Revista Chilena de Historia Natural 77: 711-723.

SISK TD, AE LAUNER, KR SWITKY \& PR EHRLICH (1994) Identifying extinction threats. BioScience 44: 592-604

STEPHENS DW \& JR KREBS (1986) Foraging theory. Princeton University Press, Princeton, NJ.

TAKIMOTO G (2003) Adaptive plasticity in ontogenetic niche shifts stabilizes consumerresource dynamics. The American Naturalist 162: 93-109.

VALDOVINOS FS, R RAMOS-JILIBERTO, JD FLORES, C ESPINOZA \& G LÓPEZ (2009) Structure and dynamics of pollination networks: The role of alien plants. Oikos 118: 1190-1200. 